\title{
The unidirectional valve patch provides no benefits to early and long-term survival in patients with ventricular septal defect and severe pulmonary artery hypertension
}

\author{
Hui-Li Gan, MD, PhD, Jian-Qun Zhang, MD, Zhao-Guang Zhang, MD, Yi Luo, MD, \\ Qi-Wen Zhou, MD, and Ping Bo, MD
}

Objective: Our aim was to test whether a unidirectional valve patch would provide benefit to early and long-term survival for patients with ventricular septal defect and severe pulmonary artery hypertension.

\begin{abstract}
Methods: Eight hundred seventy-six cases of ventricular septal defect with severe pulmonary artery hypertension were closed with or without a unidirectional valve patch and were classified as the unidirectional valve patch (UVP) group $(n=195)$ and nonvalve patch $(N V P)$ group $(n=681)$, respectively. Propensity scores of inclusion into the UVP group were used to match 138 pairs between the 2 groups. Kaplan-Meier survival curves were constructed to compare early and long-term survival.
\end{abstract}

Results: For the 138 propensity-matched pairs, there were 7 and 9 early deaths (in-hospital deaths) in the UVP and NVP groups, respectively. The difference in early mortality between the 2 groups did not reach statistical significance $\left(\chi^{2}=0.265, P=.6064\right)$. With a mean of $9.2 \pm 4.92$ years' and 2511 patient-years' follow-up, there were 6 late deaths in the UVP group and 7 late deaths in the NVP group. The difference in actuarial survival at 5 , 10,15 , and 18 years between the 2 groups was not significant $\left(\log\right.$-rank test, $\left.\chi^{2}=0.565, P=.331\right)$. The difference in the late mortality between the groups with or without a patent patch at the time of discharge did not reach statistical significance $\left(\chi^{2}=1.140, P=.2856\right)$. There was no difference between the 2 groups in the 6 -minute walk distance assessed at the last follow-up $(525.9 \pm 88.0$ meters for the UVP group and $536.5 \pm 95.8$ meters for the NVP group, $\mathrm{F}=1.550, P=.214$ ).

Conclusion: A unidirectional valve patch provides no benefits to early and long-term survival when it is used to deal with ventricular septal defect and severe pulmonary artery hypertension. (J Thorac Cardiovasc Surg 2010;139:950-5)

Severe pulmonary artery hypertension (PAH) could put patients with a large ventricular septal defect (VSD) at increased risk of morbidity and mortality when closure of the VSD is performed. Facing this situation, in some cardiac centers a unidirectional valve patch (UVP) has been used to deal with severe PAH associated with a large VSD, especially in the population affected by Eisenmenger syndrome, in an attempt to provide better early and long-term survival. To test whether a UVP for closure of a VSD benefits patients with severe PAH, we retrospectively reviewed our practice in patients who underwent the UVP procedure to close a large VSD. The present work was to compare the early and late outcomes of surgical closure of a large VSD with severe PAH with and without a UVP. A propensity score analysis was per-

From the Department of Cardiac Surgery, Beijing Anzhen Hospital, Capital Medical University, Beijing Institute of Heart, Lung and Blood Vessel Diseases, Beijing, China.

Disclosures: None.

Received for publication Nov 4, 2008; revisions received April 9, 2009; accepted for publication May 15, 2009; available ahead of print Oct 26, 2009.

Address for reprints: Hui-Li Gan, MD, PhD, Cardiac Surgery Department, Beijing Anzhen Hospital, Capital Medical University, Beijing Institute of Heart, Lung and Blood Vessel Diseases, Beijing 100029, China (E-mail: ganhuili@hotmail. com).

$0022-5223 / \$ 36.00$

Copyright (c) 2010 by The American Association for Thoracic Surgery doi:10.1016/j.jtcvs.2009.05.047 formed to match the 2 distinct groups and permit comparison and to study the association of UVP and mortality.

\section{MATERIALS AND METHODS}

The institutional review board approved this study and waived the requirement for individual consent because of its retrospective nature.

\section{Patients}

From January 1, 1990, to November 1, 2008, 1012 patients with a VSD with severe PAH were admitted to and treated in Anzhen Hospital. For 876 $(86.56 \%)$ of these patients, complete preoperative homodynamic data were available. The medical records of all 876 patients were retrospectively reviewed, and the patients were retrospectively divided into the UVP group $(n=195)$ or the nonvalve patch $(N V P)$ group $(n=681)$, respectively, according to whether a UVP was used during surgical closure of the VSD. $\mathrm{PAH}$ is defined as a mean pulmonary artery pressure (mPAP) of greater than $25 \mathrm{~mm} \mathrm{Hg}$ at rest or $30 \mathrm{~mm} \mathrm{Hg}$ with exercise, and severe PAH is further defined as an mPAP of greater than $50 \mathrm{~mm} \mathrm{Hg}$ (at rest), which is in line with the definition used by the American College of Chest Physicians. ${ }^{1}$ Pulmonary artery pressure (PAP) and mPAP and cardiac output were measured through a right heart catheterization. All patients were subjected to a $10-\mathrm{min}$ ute inhalation of pure oxygen and a 10-minute inhalation of $30 \mathrm{ppm}$ of nitric oxide (NO), respectively, during right heart catheterization. A decrease in pulmonary vascular resistance (PVR) of greater than 6.5 Wood's units (WU) from baseline was considered a positive reaction for pure oxygen or NO challenge. If a patient had a bidirectional shunt on echocardiographic analysis and a total PVR of greater than $10 \mathrm{WU}$, a pulmonary vascular resistance index (PVRI) of greater than $6 \mathrm{WU} / \mathrm{m}^{2}$, a pulmonary/systemic flow 


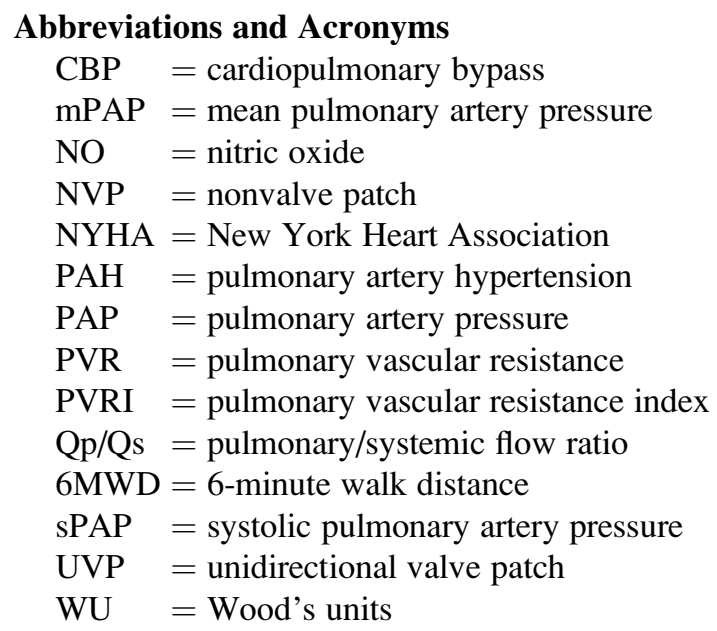

ratio (Qp/Qs) of less than 1.5, or a hemoglobin value of greater than $160 \mathrm{~g} / \mathrm{L}$, he or she would be more likely assigned to the UVP group. The age at which the patients were admitted to Anzhen Hospital ranged from 2 to 37 years. The preoperative demographics and risk factors of patients from the 2 groups are presented in Table 1. Patients in the UVP group were older and statistically more likely to have a history of cyanosis on exertion $(P=.0001)$, refractory right heart failure $(P=.0003)$, a hemoglobin value of $160 \mathrm{~g} / \mathrm{L}$ or greater $(P=.001)$, a bidirectional shunt on echocardiographic analysis $(P=0.0001)$, a systolic pulmonary artery pressure (sPAP) of 110 $\mathrm{mm} \mathrm{Hg}$ or greater $(P=.007)$, a pulmonary/systemic systolic artery pressure ratio of 0.9 or greater $(P=.0324)$, higher PVR $(P=.019)$, higher PVRI $(P=.015)$, higher mPAP $(P=0.040)$, lower Qp/Qs $(P=.0001)$, lower $\mathrm{PaO}_{2}(P=.0009)$, lower arterial oxygen saturation $(P=.017)$, shorter 6-minute walk distance (6MWD; $P=.0003$ ), or less chance of a positive reaction to NO $(P=.0112)$ or pure oxygen $(P=.0001)$ inhalation during the right heart catheterization than were the patients in the NVP group.

\section{Therapeutic Regimen}

Before the procedure, a Swan-Ganz pulmonary artery catheter was inserted for all patients, and PAP was monitored throughout the perioperative period. All patients underwent operations with general anesthesia and moderate hypothermic cardiopulmonary bypass (CPB) with cardiac arrest. After the $\mathrm{CPB}$ was established through cannulation in the superior and inferior venae cavae and ascending aorta, the ascending aorta was clamped, and crystalloid cardioplegia was perfused. The VSD was closed by means of a standard procedure with a Dacron patch in the NVP group, whereas a UVP was used to close the VSD in the UVP group, as previously described. ${ }^{2}$ In brief, the UVP was constructed from a Dacron patch approximately as large as the defect to be closed. A hole approximately 0.5 to $1.0 \mathrm{~cm}$ in diameter was made in the patch somewhat off center. A piece of quadrangular pericardium was attached by sutures to the surface of the Dacron patch. The 3 edges of pericardium were continuously sutured, and 1 edge was left unattached. This unattached edge of pericardium should provide a small opening not to exceed $0.5 \mathrm{~cm}$, and appropriate tension should be maintained on the pericardial edge so that the valve mechanism will not be loose and incompetent. The CPB duration was $95.2 \pm 24.3$ minutes, and the clamping time was $34.4 \pm 18.3$ minutes. The nasopharyngeal temperature during $\mathrm{CPB}$ was $28.5^{\circ} \mathrm{C} \pm 5.5^{\circ} \mathrm{C}$. During the follow-up period, $48 \%$ of the cohort were treated with a $\beta$-blocker, $78 \%$ were treated with an angiotensin-converting enzyme inhibitor, $59 \%$ were treated with a calcium channel blocker (diltiazem), $42 \%$ were treated with a cardiotonic glycoside, $38 \%$ were treated with a diuretic, and $19 \%$ were treated with potassiumsparing diuretics, such as spironolactone or amiloride. Six percent of pa- tients were anticoagulated with warfarin, whereas $23 \%$ of patients were treated with antiarrhythmic drugs. In addition, $25 \%$ of patients had oxygen therapy at home, and $3 \%$ of patients were treated with advanced therapies for PAH using bosentan (a dual endothelin receptor antagonist), sildenafil (a phosphodiesterase-5 inhibitor), or a combination of both.

\section{Follow-up}

Of the 876 patients, valid and complete follow-up information was obtained from 843 (96.2\%) through mail, e-mail, telephone calls, or outpatient department visits. Long-term clinical outcome was retrospectively assessed up to November 2008 through telephone interviews of the patients using a standard questionnaire and by reviewing the medical records of their cardiologists, general practitioners, or both when appropriate. All patients were asked to return to Anzhen Hospital or a local hospital for an echocardiogram and clinical evaluation. During the follow-up period, PAP was measured through echocardiographic analysis, and the 6MWD was also assessed. Baseline demographics, procedural data, and perioperative outcomes were recorded. The mean follow-up period for the whole cohort was $102.2 \pm 61.6$ months.

\section{Statistical Analysis}

All statistical analyses were performed retrospectively with SAS for Windows version 8.2 (SAS Institute, Inc, Cary, NC). Continuous variables are presented as means \pm standard deviations and were analyzed with Student's $t$ test. Categorical data are presented as total numbers and relative frequencies. Comparisons between the 2 groups were made by using the Mann-Whitney $U$ test or the $\chi^{2}$ test, as appropriate. Kaplan-Meier survival curves were constructed to illustrate the actuarial survival. Comparisons of time-related data are made by using the log-rank test. In an attempt to control for selection bias, propensity scores were estimated by using unconditional logistic regression to determine the predicted probability of inclusion into the UVP group for each of the 876 patients.

First, multivariate logistic regression was used to identify the characteristics with which surgical closure of a VSD with a UVP was liable to be selected. Of 27 defining baseline variables, 8 were not significant in the logistic regression analysis. These were sex, left ventricular ejection fraction, cardiac/thoracic ratio, electrocardiographic rhythm, New York Heart Association (NYHA) functional class, cyanosis at rest, hemoptysis, and clubbing of fingers and toes. Logistic regression analysis identified 10 variables as significant predictors of UVP use. These 10 variables were a PAP of greater than $110 \mathrm{~mm}$ $\mathrm{Hg}$, a PVR of greater than $10 \mathrm{WU}$, a PVRI of greater than $6 \mathrm{WU} / \mathrm{m}^{2}$, a Qp/Qs of less than 1.5 , a hemoglobin value of greater than $160 \mathrm{~g} / \mathrm{L}$, a negative reaction to $\mathrm{NO}$ and pure oxygen inhalation in the right heart catheterization (defined as a PVR decrease $>6.5 \mathrm{WU}$ after inhalation of $\mathrm{NO}$ and pure oxygen), bidirectional shunt on ultracardiographic analysis, a systolic heart murmur of less than grade III out of 6 grades, and an arterial oxygen saturation of less than $92 \%$ on air.

Second, a propensity score was calculated for all 876 patients using these significant regression coefficients. Each patient in the UVP group was then closely matched with an NVP group patient having the same or nearest propensity score (with the same propensity score \pm 0.05 , the approach is to match UVP and NVP subjects on the first 5 digits of the propensity score). The matching program was performed in the total population and has successfully matched 138 patients in the UVP group with 138 patients in the NVP group. The resulting 138 pairs of matched patients are the focus of the outcome analysis in this study.

\section{RESULTS}

\section{Entire Unmatched Patient Population}

During the in-hospital stay, there were $11(5.64 \%)$ and 41 $(6.02 \%)$ early deaths in the UVP and NVP groups, respectively. Of the 52 early deaths, 47 were due to PAH crisis and acute right heart failure, 3 were due to reperfusion injury, 
TABLE 1. Preoperative baseline demographic variables and risk factors in the UVP and NVP groups

\begin{tabular}{|c|c|c|c|}
\hline Demographics and preoperative risk factors & UVP group $(n=195)$ & NVP group $(n=681)$ & $P$ value $\left(\chi^{2}\right)$ \\
\hline Age (y) & $25.76 \pm 8.51$ & $18.40 \pm 8.13$ & $.028(5.96)$ \\
\hline Female sex $(\%)$ & 35.90 & 40.96 & $.202(1.627)$ \\
\hline Right heart failure ( $\%)$ & 18.46 & 12.04 & $.0003(13.359)$ \\
\hline Cyanosis at rest $(\%)$ & 5.64 & 4.70 & $.591(0.288)$ \\
\hline Cyanosis on exertion $(\%)$ & 40.00 & 27.17 & $.0006(11.885)$ \\
\hline Hemoglobin $\geq 160 \mathrm{~g} / \mathrm{L}(\%)$ & 34.87 & 22.91 & $.001(10.838)$ \\
\hline Clubbing of fingers and toes $(\%)$ & 24.10 & 18.65 & $.092(2.832)$ \\
\hline Systolic heart murmur $\geq \mathrm{II} / 6$ grade $(\%)$ & 65.94 & 78.26 & $.0225(5.206)$ \\
\hline $6 \mathrm{MWD}<400 \mathrm{~m}(\%)$ & 27.69 & 16.15 & $.0003(13.266)$ \\
\hline LVEF & $0.55 \pm 0.07$ & $0.61 \pm 0.06$ & $.082(2.223)$ \\
\hline Bidirectional shunt on UCG $(\%)$ & 73.39 & 46.98 & $.0001(43.890)$ \\
\hline PVR (WU) & $15.9 \pm 6.31$ & $12.06 \pm 6.42$ & $.019(5.87)$ \\
\hline PVRI (WU/m²) & $10.6 \pm 4.21$ & $8.40 \pm 4.48$ & $.015(6.32)$ \\
\hline QP/Qs $<1.5(\%)$ & 46.67 & 27.31 & $.0001(26.261)$ \\
\hline $\mathrm{sPAP} \geq 110 \mathrm{~mm} \mathrm{Hg}(\%)$ & 49.23 & 38.47 & $.007(7.260)$ \\
\hline $\mathrm{sPAP} / \mathrm{sBP}$ ratio $\geq 0.90$ & $0.97 \pm 0.18$ & $0.87 \pm 0.145$ & $.0324(4.974)$ \\
\hline mPAP (mm Hg) & $81.48 \pm 17.21$ & $69.73 \pm 15.2$ & $.040(3.87)$ \\
\hline $\mathrm{SaO}_{2}$ & $0.82 \pm 0.15$ & $0.93 \pm 0.05$ & $.017(6.890)$ \\
\hline $\mathrm{PaO}_{2}<60 \mathrm{~mm} \mathrm{Hg}(\%)$ & 22.56 & 12.92 & $.0009(11.012)$ \\
\hline Hematoasthenia pulmonary field on $\mathrm{x}$-ray film (\%) & 26.67 & 18.94 & $.0188(5.516)$ \\
\hline CTR & $0.63 \pm 0.064$ & $0.62 \pm 0.069$ & $.323(0.977)$ \\
\hline Sinus rhythm on ECG $(\%)$ & 94.36 & 95.74 & $.415(0.665)$ \\
\hline RBBB $(\%)$ & 35.89 & 27.90 & $.0311(4.645)$ \\
\hline Hemoptysis $(\%)$ & 4.62 & 3.38 & $.417(0.660)$ \\
\hline NYHA functional class $\geq$ III $(\%)$ & 18.46 & 17.77 & $.824(0.05)$ \\
\hline Positive reaction to NO inhalation (\%) & 39.49 & 49.78 & $.0112(6.440)$ \\
\hline Positive reaction to pure oxygen inhalation (\%) & 43.59 & 61.23 & $.0001(19.288)$ \\
\hline
\end{tabular}

$\overline{U V P}$, Unidirectional valve patch; $N V P$, nonvalve patch; $6 M W D, 6$-minute walk distance; $L V E F$, left ventricular ejection fraction; $U C G$, ultracardiography; $P V R$, pulmonary vascular resistance; $W U$, Wood's units; $P V R I$, pulmonary vascular resistance index; $Q p / Q s$, pulmonary/systemic flow ratio; $s P A P$, systolic pulmonary artery pressure; $s P A P / s B P$, pulmonary/systemic systolic artery pressure ratio; $m P A P$, mean pulmonary artery pressure; $\mathrm{SaO}_{2}$, arterial oxygen saturation; $C T R$, cardiac/thoracic ratio; $E C G$, echocardiography; $R B B B$, right bundle branch block; $N Y H A$, New York Heart Association; $N O$, nitric oxide.

and 2 were due to lung infection. According to the $\chi^{2}$ test, the difference in the early mortality between the UVP and NVP groups did not reach statistical significance $\left(\chi^{2}=0.001\right.$, $P=.973)$. With a mean of $102.2 \pm 61.6$ months and 7969 patient-years' follow-up, there were 10 late deaths in the UVP group and 26 late deaths in the NVP group. Of the total 36 cases of late death, 27 were due to refractory chronic right heart failure, 3 were due to PAH crisis, 4 were due to copious hemoptysis, and 2 were due to severe lung infection.

\section{Propensity-Matched Pairs $(\mathbf{n}=\mathbf{1 3 8})$}

All characteristics that differed significantly between the 2 groups before matching became comparable (Table 2). During the in-hospital stay, there were $7(5.07 \%)$ and $9(6.52 \%)$ early deaths for the UVP and NVP groups, respectively. Of the 16 early deaths, 14 were due to PAH crisis, and 2 were due to reperfusion injury. According to the $\chi^{2}$ test, the difference in the early mortality between the UVP and NVP groups did not reach statistical significance $\left(\chi^{2}=0.265\right.$, $P=.6064)$. Intraoperative epicardial or transesophageal echocardiographic analysis showed the UVP open and blood flowing from the right to the left ventricle in all of the UVP group patients. The UVP gradually closed with improve- ment of hemodynamics in the UVP group, as evidenced with serial echocardiographic analysis, and thus by the third postoperative day, the UVP had closed in 118 patients and remained only slightly open in 20 patients. At 72 hours after the VSD closure procedure, the mPAP assessed with a Swan-Ganz pulmonary artery catheter was $49.7 \pm 29.6$ $\mathrm{mm} \mathrm{Hg}$ for the UVP group and $52.2 \pm 28.1 \mathrm{~mm} \mathrm{Hg}$ for the NVP group $(t=0.7196, P=.4724)$.

With a mean of $9.2 \pm 4.92$ years' and 2511 patient-years' follow-up, there were 6 late deaths in the UVP group and 7 late deaths in the NVP group. Among the 13 total cases of late death, 10 were due to refractory chronic right heart failure, 1 was due to PAH crisis, and 1 was due to copious hemoptysis. As shown in Figure 1 and Table 3, the Kaplan-Meier survival curves were estimated to compare the long-term actuarial survival between the 2 groups in the 138 propensity score-matched pairs. The actuarial survival at $5,10,15$, and 18 years of the 2 groups was not significantly difference (log-rank test, $\chi^{2}=0.565, P=.331$ ). During the follow-up period, of the 20 patients known to have a patent patch at the time of discharge, there were 2 late deaths, whereas of the 240 patients (including 129 in the NVP group and 111 in the UVP group known to have a closed patch at the 
TABLE 2. Preoperative baseline demographic variables and risk factors of the propensity-matched patients

\begin{tabular}{|c|c|c|c|}
\hline Demographics and preoperative risk factors & UVP group $(n=138)$ & NVP group $(n=138)$ & $P$ value $\left(\chi^{2}\right)$ \\
\hline Age (yr) & $25.43 \pm 9.32$ & $24.42 \pm 8.76$ & $.082(4.76)$ \\
\hline Female sex $(\%)$ & 32.60 & 31.88 & $.898(0.017)$ \\
\hline Right heart failure $(\%)$ & 15.22 & 14.49 & $.866(0.029)$ \\
\hline Cyanosis at rest $(\%)$ & 15.9 & 13.8 & $.612(0.258)$ \\
\hline Cyanosis on exertion $(\%)$ & 38.41 & 32.60 & $.314(1.013)$ \\
\hline Hemoglobin $\geq 160 \mathrm{~g} / \mathrm{L}(\%)$ & 28.99 & 25.36 & $.499(0.458)$ \\
\hline Clubbing of fingers and toes $(\%)$ & 23.91 & 19.57 & $.381(0.767)$ \\
\hline Systolic heart murmur $\geq \mathrm{II} / 6$ grade $(\%)$ & 72.46 & 78.26 & $.264(1.249)$ \\
\hline $6 \mathrm{MWD}<400 \mathrm{~m}(\%)$ & 23.19 & 21.01 & $.689(0.163)$ \\
\hline LVEF & $0.55 \pm 0.07$ & $0.60 \pm 0.07$ & $.082(2.223)$ \\
\hline Bidirectional shunt on UCG $(\%)$ & 72.46 & 65.94 & $.241(1.377)$ \\
\hline PVR (WU) & $13.4 \pm 4.54$ & $12.72 \pm 4.67$ & $.319(1.87)$ \\
\hline PVRI (WU/m²) & $8.65 \pm 4.07$ & $8.48 \pm 3.51$ & $.332(1.65)$ \\
\hline $\mathrm{QP} / \mathrm{Qs}<1.5(\%)$ & 52.17 & 44.93 & $.228(1.450)$ \\
\hline $\mathrm{sPAP} \geq 110 \mathrm{~mm} \mathrm{Hg}(\%)$ & 50 & 46.38 & $.547(0.363)$ \\
\hline $\mathrm{sPAP} / \mathrm{sBP}$ & $0.97 \pm 0.18$ & $0.96 \pm 0.165$ & $.624(0.874)$ \\
\hline mPAP $(\mathrm{mm} \mathrm{Hg})$ & $76.48 \pm 17.21$ & $75.81 \pm 16.4$ & $.19(1.87)$ \\
\hline $\mathrm{SaO}_{2}$ & $0.82 \pm 0.15$ & $0.85 \pm 0.05$ & $.270(1.045)$ \\
\hline $\mathrm{PaO}_{2}<60 \mathrm{~mm} \mathrm{Hg}(\%)$ & 21.74 & 19.57 & $656(0.199)$ \\
\hline Hematoasthenia lung field on $\mathrm{x}$-ray film $(\%)$ & 27.54 & 22.46 & $.331(0.947)$ \\
\hline CTR & $0.63 \pm 0.064$ & $0.62 \pm 0.069$ & $.323(0.977)$ \\
\hline Sinus rhythm on ECG $(\%)$ & 93.48 & 96.38 & $.274(1.04)$ \\
\hline RBBB $(\%)$ & 35.51 & 34.06 & $.801(0.064)$ \\
\hline Hemoptysis $(\%)$ & 4.38 & 2.90 & $.520(0.415)$ \\
\hline NYHA functional class $\geq \mathrm{III}(\%)$ & 19.57 & 15.94 & $.431(0.620)$ \\
\hline Positive reaction to NO inhalation $(\%)$ & 49.28 & 57.97 & $.148(2.098)$ \\
\hline Positive reaction to pure oxygen inhalation $(\%)$ & 50.72 & 60.87 & $.090(2.879)$ \\
\hline
\end{tabular}

See Table 1 for a list of abbreviations.

time of discharge), there were 11 late deaths. The difference in the late mortality between the groups with and without a patent patch at the time of discharge did not reach statistical significance $\left(\chi^{2}=1.140, P=.2856\right)$. Echocardiographic analysis at follow-up showed that the UVP had closed in 121 of the 125 survivors in the UVP group, whereas the other 4 had a 1- to 3-mm shunt across the UVP. Echocardiographic analysis found severe tricuspid regurgitation in 9 UVP group patients and in $11 \mathrm{NVP}$ group patients $\left(\chi^{2}=0.274, P=\right.$ .6008). The sPAP assessed through echocardiographic analysis was $56.7 \pm 21.1 \mathrm{~mm} \mathrm{Hg}$ for the UVP group and $58.2 \pm$ $19.3 \mathrm{~mm} \mathrm{Hg}$ for the NVP group $(t=0.4625, P=.6441)$. The 6MWDs assessed at the last follow-up were $525.9 \pm$ 88.0 meters for the UVP group and $536.5 \pm 95.8$ meters for the NVP group, according to the $t$ test, and the difference between the 2 groups did not reach statistical significance ( $t=$ $0.9060, P=.3658)$. Of the 125 survivors in the UVP group, there were 8 in NYHA functional class III to IV, and of the 122 survivors in the NVP group, there were 9 in NYHA functional class III to IV $\left(\chi^{2}=0.7617, P=.092\right)$.

\section{DISCUSSION}

The closure of a VSD is usually performed at an early age and before the onset of increased PVR in most industrialized countries. Although patients with a large VSD and increased
PVR are rarely seen in surgically advanced countries, they continue to represent a significant proportion of the congenital heart disease population in underdeveloped countries. Delayed presentation and operations for patients with VSD and PAH are not uncommon in the developing world. ${ }^{3,4}$ The closure of VSD in patients with a PVR of $10 \mathrm{WU}$ or greater is considered to be contraindicated because of high operative mortality and debatable long-term results. ${ }^{5}$ These patients often have a turbulent postoperative course as the result of PAH. A large VSD exposes the patients to the risk of $\mathrm{PAH}$ and tends to worsen with age. The course of the disease is variable and depends on the size of the defect, the magnitude of the left-to-right shunt, and the pulmonary vascular response to increased pulmonary flow and pressure. ${ }^{6}$

Pulmonary hypertensive crisis can be associated with acute congestive heart failure, which is the main cause of death. ${ }^{7-10}$ To prevent acute right ventricular failure from refractory PAH and early postoperative death, a wide variety of UVPs have been described. ${ }^{11}$ The UVP technique provides a simple physiologic mechanism for unloading the right ventricle during periods of severe, acute, and transient or sustained PAH. In 1959, Bailey and colleagues first applied flap valves made of a compressed ring of polyvinyl sponge in 8 patients with atrial and ventricular septal shunts. The conventional closure procedure appeared to be too risky 


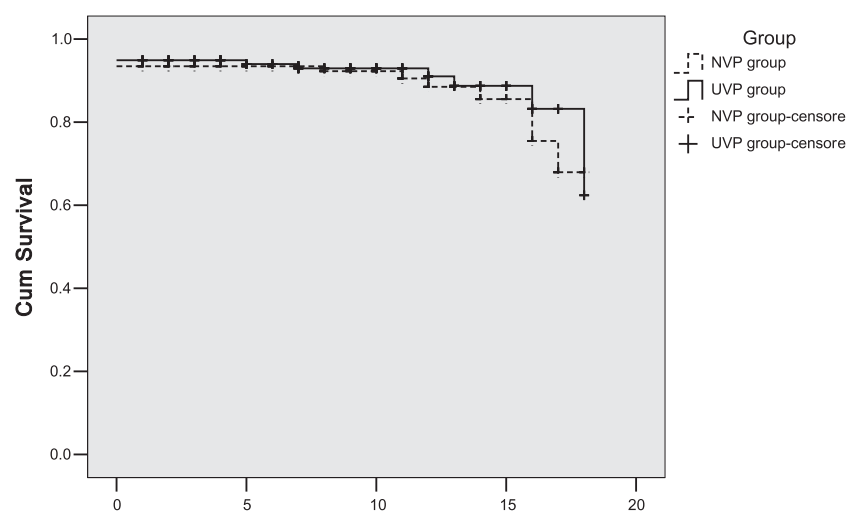

FIGURE 1. The Kaplan-Meier survival curve for the unidirectional valve patch $(U V P)$ and nonvalve patch (NVP) groups (survival years).

because of the presence of severe PAH in these patients. Seven of the 8 patients with Bailey's device survived. There was a gradual decrease in PAP and eventually total or near total closure of the defect. ${ }^{12,13}$ The UVP was also described by Ad and associates ${ }^{14}$ who adopted a 1-way, valved, atrial septal patch in 18 patients. Then Zhou and coworkers ${ }^{15}$ adopted a similar UVP in the treatment of VSD complicated with Eisenmenger syndrome, and Zhang and colleagues ${ }^{16}$ developed a unidirectional monovalve homologous aortic patch for repair of VSD with PAH. Novick and associates ${ }^{17}$ reported the use of a nonfenestrated patch closure for children younger than 6 years with severe PAH, but the operative mortality was very high. In recent years, creation of an intracardiac shunt to prevent right ventricular failure has been used with success. ${ }^{18}$

Although the fabrication method of these UVPs was not exactly the same, the rationale for them was practically identical, which permits a right-to-left shunt to relieve right heart overload and left heart hypovolemia and helps the patient survive the PAH crisis. Kannan and coworkers ${ }^{19}$ reported that the closure of a large VSD in patients with increased PVR was warranted, and the early mortality rate in their studies using a nonfenestrated patch was $13.1 \%$. Novick and associates ${ }^{17}$ reported a study in a series of 91 children with a large VSD and increased PVR who underwent double-patch VSD closure with an early mortality of 3.6\%. Although this mortality rate is much lower than that seen in Kannan and coworkers' study, ${ }^{19}$ this comparison was not warranted because the children in the Kannan and coworkers' study were older (7.5 years) than those in Novick and associates' study ${ }^{17}$ (4.0 years), and the patients' PVRs in the 2 studies were not comparable. We believe that this difference could not provide evidence to support the hypothesis that the double-flap valve VSD closure technique produces a lower mortality rate among patients with PAH and increased PVR.

The purpose of surgical repair of the congenital systemicto-pulmonary shunts with severe PAH is to achieve a higher
TABLE 3. The actuarial survival of the UVP and NVP groups for the 138 propensity-matched pairs $(\%)$

\begin{tabular}{lllr}
\hline & UVP group & NVP group & $\boldsymbol{P}$ value* $\left(\chi^{2}\right)$ \\
\hline 5-y Survival & $94.0 \pm 2.1$ & $93.5 \pm 2.1$ & $.565(0.331)$ \\
10-y Survival & $93.0 \pm 2.3$ & $90.5 \pm 2.9$ & \\
15-y Survival & $88.8 \pm 3.6$ & $85.6 \pm 4.4$ & \\
18-y Survival & $62.4 \pm 18.6$ & $67.9 \pm 10.0$ &
\end{tabular}

$U V P$, Unidirectional valve patch; $N V P$, nonvalve patch. *Log-rank test of KaplanMeier survival curve between the UVP and NVP groups.

survival rate with excellent quality of life. ${ }^{20}$ It would not be considered beneficial when the patients only obtain operational success but not a higher long-term survival rate with excellent quality of life. The debate about the adoption of the UVP is whether the UVP would provide benefits to early and long-term survival for the closure of systemic-to-pulmonary shunts with severe PAH. It is difficult to reach a conclusion from the results with various techniques because of the small numbers in the existing studies and the heterogeneity of the patient population and because of the absence of a randomized study with control subjects or a series with literature controls at the point of the hierarchy of evidence. From our analysis, of the 138 propensity-matched pairs, there were no significant differences in the in-hospital mortality or actuarial survival at 5, 10, 15, and 18 years and in 6MWDs at the last follow-up between the 2 groups. To investigate whether a patent patch at the time of discharge would provide further benefits for long-term survival, we compared the long-term survival of the patients known to have a fenestrated patch with that of those who do not have a fenestrated patch or have a closed patch at the time of discharge. Again, the difference in the late mortality between the groups with and without a patent patch at the time of discharge did not reach statistical significance. Thus we conclude that the UVP provides no benefits to early and long-term survival when it is used to repair a VSD with severe PAH.

This study is a retrospective analysis, and therefore some confounding factors might be involved to show any clear relationship between the early and late prognosis and the use of the UVP in the closure of a VSD with severe PAH. Despite the relatively large number of VSD and UVP cases in our study compared with previously published reports and the propensity score analysis used in the present work, which allowed us to balance the 2 nonequivalent groups and reduce significant bias for subclassification of the patients, a prospective randomized study is needed to provide more substantial evidence for our conclusion.

\footnotetext{
References

1. Rubin LJ. American College of Chest Physicians. Diagnosis and management of pulmonary arterial hypertension: ACCP evidence-based clinical practice guidelines. Chest. 2004;126(suppl):4S-6S.

2. Zhou Q, Lai Y, Wei H, Song R, Wu Y, Zhang H. Unidirectional valve patch for repair of cardiac septal defects with pulmonary hypertension. Ann Thorac Surg. 1995;60:1245-8.
} 
3. Saxena A. Congenital heart disease in India. A status report. Ind J Pediatr. 2005; 72:595-8.

4. Anonymous. Health manpower requirements for the achievement of health for all by the year 2000 through primary health care. Report of a WHO Expert Committee. World Health Organ Tech Rep Ser. 1985;717:7-92.

5. de Leval M. Ventricular septal defects. In: Stark J, de Leval M, eds. Surgery for congenital heart defects. Philadelphia: WB Saunders; 1994.p.355-72.

6. Wells WJ, Lindesmith GG. Ventricular septal defect. In: Arciniegas E, ed. Pediatric cardiac surgery. Chicago: Year Book Medical; 1985.p.141-53.

7. Rich S, Kaufmann E, Levy PS. The effect of high doses of calcium-channel blockers on survival in primary pulmonary hypertension. $N$ Engl J Med. 1992;327:76-81.

8. Hallidie Smith KA, Hollman A, Cleland WP, Bentall HH, Goodwin JF. Effects of surgical closure of ventricular septal defects upon pulmonary vascular disease. $\mathrm{Br}$ Heart J. 1969;31:246-60.

9. Fried R, Falkovsky G, Newburger J, et al. Pulmonary arterial changes in patients with ventricular septal defects and severe pulmonary hypertension. Pediatr Cardiol. 1986;7:147-54.

10. Rame JE. Pulmonary hypertension complicating congenital heart disease. Curr Cardiol Rep. 2009;11:314-20.

11. Choudhary SK, Talwar S, Airan B. A simple technique of unidirectional valved patch for closure of septal defects. J Thorac Cardiovasc Surg. 2007;134:1357-8.

12. Robicsek F. Flap-valve closure of ventricular septal defects. Ann Thorac Surg. 2006;81:788
13. Larios R, Fitch EA, Blanco G, Bailey CP. The use of an artificial foraminal valve prosthesis in the closure of interatrial and interventricular septal defects. Dis Chest. 1959;36:631-41.

14. Ad N, Birk E, Barak J, Diamand S, Snir E, Vidne BA. A one way atrial septal patch: a new surgical technique and its clinical application. J Thorac Cardiovasc Surg. 1996;111:841-8.

15. Zhou Q, Lai Y, Wei H, Song R, Wu Y, Zhang H. Unidirectional valve patch for repair of cardiac septal defects with pulmonary hypertension. Ann Thorac Surg. 1995;60:1245-8

16. Zhang B, Wu S, Liang J, et al. Unidirectional monovalve homologous aortic patch for repair of ventricular septal defect with pulmonary hypertension. Ann Thorac Surg. 2007;83:2176-81.

17. Novick WM, Sandoval N, Lazorhysynets VV, et al. Flap valve double patch closure of ventricular septal defects in children with increased pulmonary vascular resistance. Ann Thorac Surg. 2005;79:21-8.

18. Novick WM, Gurbuz AT, Watson DC, et al. Double patch closure of ventricula septal defect with increased pulmonary vascular resistance. Ann Thorac Surg. 1998;66:1533-8.

19. Kannan BR, Sivasankaran S, Tharakan JA, et al. Long-term outcome of patients operated for large ventricular septal defects with increased pulmonary vascular resistance. Ind Heart J. 2003;55:161-6.

20. Beat F, Langford K, William T, et al. Ventricular Septal defect with increased pulmonary vascular resistance. Am J Cardiol. 1974;33:403-9. 